\title{
THE NOAA SATELLITE OBSERVING SYSTEM ARCHITECTURE STUDY
}

\author{
Dr. Stephen Volz, NOAA/NESDIS \\ Dr. Mark Maier, The Aerospace Corporation \\ Mr. David Di Pietro, NASA/Goddard Space Flight Center
}

\begin{abstract}
NOAA is beginning a study, the NOAA Satellite Observing System Architecture (NSOSA) study, to plan for the future operational environmental satellite system that will follow GOES and JPSS, beginning about 2030. This is an opportunity to design a modern architecture with no preconceived notions regarding instruments, platforms, orbits, etc., but driven by user needs. The NSOSA study team will develop and evaluate architecture alternatives, to include partner and commercial alternatives that are likely to become available. The objectives will include both functional needs and strategic characteristics (e.g., resiliency, flexibility, responsiveness, sustainability). The study will be informed by the Space Platform Requirements Working Group (SPRWG), commissioned by NESDIS. The SPRWG is charged to assess new or existing user needs and to provide relative impacts from different candidate observing systems in the future architecture. SPRWG results will serve as input to the process for new foundational (Level 0 and Level 1) requirements for the next generation of NOAA satellites that follow the GOES-R, JPSS, DSCOVR, Jason-3, and COSMIC-2 missions.
\end{abstract}

\section{INTRODUCTION}

Over the next few years the United States will begin operating a new generation of weather, space weather, and environmental remote sensing satellites. These include the JPSS low Earth orbit (LEO) satellites, and the GOES-R series of geostationary (GEO) satellites, as well as DSCOVR, Jason-3, and the COSMIC-2 constellation. This Program of Record (POR) will supply essential weather and environmental satellite services into the late 2020 s or somewhat beyond, depending on the particular service. Given the newness of these capabilities it might seem premature that NOAA is already pursuing serious planning for the generation after these systems. But examination of the most likely lifetimes of the current generation of systems and typical development timelines for major satellite programs shows that it is far from too early. Opportunities exist to effect next generation systems within two years. If change is needed, it will be necessary to make major decisions on a similar timeframe.
In 2013, under the "Strengthening NESDIS" initiative, the newly formed Office of Systems Architecture and Advanced Planning within NOAA/NESDIS began preliminary studies of the post-POR satellite architecture. These studies identified the need to begin making decisions about the next generation satellite systems within a few years, identified priority areas within the problem and solution spaces needing further study, and examined whether or not significant departures from the POR architecture could have significant cost or performance benefits. On the basis of these preliminary studies, NOAA/NESDIS has initiated a formal study of the next generation weather and environmental remote sensing satellite architecture.

\section{THE NEXT GENERATION PROBLEM}

The study problem is concisely stated as:

Determine the most cost effective space segment architectures for performing NOAA weather, space weather, and environmental remote sensing missions (excluding land mapping), beyond the POR to 2050. Architecture alternatives should be compatible with an estimated annual capped NESDIS top line budget.

Behind this simple statement we recognize a number of essential considerations. An architecture problem can be best thought of as identifying, and making, the early (preconcept) decisions about future satellite systems with the largest impact on value, cost, and risk [1], [2]. It is also necessary to know when those decisions have to be made and any structure of linked decisions. It is desirable to choose architectures that enable basic decisions about allocation of functions to orbits and instrument capability to be made early and that enable implementation decisions to be deferred.

From the past study of weather satellite constellations we know that value and cost are primarily determined by choice of instruments, distribution of instruments over orbits, and replenishment policies. Development risk is determined primarily by instrument technology maturity. Operational risk, primarily the risk of capability gaps or asset wastage, is determined mostly by constellation management policy. The consequence is that our definition of a next generation architecture must encompass instrument capabilities and 
enabling technologies, allocation to orbital platforms, and launch and replenishment policy.

NESDIS has also identified other key problem-space areas that must be addressed in the study.

- How do we reconcile the need to minimize the probability of service gaps with the need to efficiently use all expensive satellite resources and rapidly bring new capabilities into operations rather than have them sitting in spare positions?

- How do we design a system that is accepting of new observing technology while still delivering consistent information content to a broad customer base?

- How will the optimal balance between data gathered for exploitation by human forecasters and data assimilated into numerical models change over the next $30+$ years?

- How do we balance mutually exclusive collection needs from a disparate stakeholder base, such as space weather from distant orbits like the L1 halo versus terrestrial weather from LEO?

- How do we balance the desire for flexibility to accommodate varying budget levels against the desire to maximize efficient use of currently projected budgets?

The study is deliberately scoped to the architecture of the satellite portion of the NESDIS enterprise and excludes the ground segment. The differing timelines for decisions and implementations between space and ground segments make it acceptable to separate the studies. While separate, the cross-dependence of the satellite architecture on architectural decisions in the ground segment and in the larger weather enterprise are understood and provisions for accounting for them are in the study plan.

\section{ARCHITECTURE STUDY APPROACH}

The NSOSA study is organized into four major tracks built around three major cycles. All tracks contribute to all cycles, and all results are consolidated into incremental products in each cycle. The four tracks are Instrument Catalog development, Environmental Data Record (EDR) Value Model development, Mission Value Model development, and Integration. Each of the cycles is referred to as a "design cycle" and each design cycle does complete, end-to-end designs of multiple alternative architectures. The work of the four tracks is supported and connected to the stakeholder community through a number of outreach efforts. The most important of these is the Space Platform Requirements Working Group (SPRWG). In addition the study will engage with a broad range of stakeholders through established forums and an advisory group.

\subsection{Instrument Catalog Development}

Past studies and experience have shown that the cost and performance of the instruments is the principal value and cost key to the weather and environmental satellite architecture. The role in value is obvious, the whole point of flying the satellites is to fly the instruments and collect their data. The capabilities of the instruments translates directly into the capabilities of the enterprise. The cost of instruments typically drives the cost of a satellite. For typical weather satellites, neither the cost of launch nor the cost of the satellite bus plays as large a role as the cost of instrument.

Because instruments play such a large role in the architecture, we have set up one major track of the study to investigate future instrument options. This task is:

- Cataloging existing and developmental instruments relevant to the NSOSA mission areas.

- Soliciting instrument concepts for the 2030 era from industry, academia, and from government laboratories.

- Selectively commissioning design studies for categories of instruments where the first two task elements yield insufficient results.

It is important to understand that the purpose of this task is not to pick any particular instrument concept for next generation satellites. Choosing a specific instrument is a task for later, after completion of the NSOSA study, when we have actually established next generation satellite acquisition programs. The purpose of the task is to identify the likely capability-size-cost relationships for instruments relevant to the mission that can be flown on satellites starting circa 2030. Where those capability-cost-size relationships become key to favorable architectures, the study will use this to identify recommended investments in sensor technology and concept development over the next few years.

\subsection{EDR Value Model Development}

The heart of problem space analysis in an architecture study is development of a value model. The NSOSA study team will develop two value models, for reasons to be described. The NSOSA study needs a value model that is much more than collection of projected requirements (note while the term "requirements" is used here, these "requirements" are not baselined and are more appropriately thought of as user needs) that can, or can't, be satisfied by an alternative. The ground rules of the study require that we be able to assess alternatives over a tradeable range that includes, but is not limited to, the projected long-term budget constraint.

In order for a value model to be useful in the study it must encompass a number of possibly contradictory constraints. 
- It needs to be of sufficient fidelity that we can trust its results in making important strategic investment decisions.

- It needs to be simple, and automated enough to allow for screening assessments of many alternatives (possibly 100's).

- It needs to accommodate assessment of architecture alternatives differing substantially from today's architecture.

We've chosen to address these issues by developing two, interrelated value models. The first, the EDR Value Model (EVM) is based on technical measures applied at a generic sensor level. The EDRs that are the subject of most of the objectives in the EVM are generics or representative of classes of existing specific EDRs. This model defines a set of objectives spanning EDR delivery, user communication services (e.g., data rebroadcast, sensor data collection), and strategic priorities. Each has a measurement scale with determined lower and upper bounds. Alternatives with performance within the bounds are in the "tradeable range" and are comparatively rated. The study team works closely with the SPRWG in building this model.

The second leg of value modeling is the Mission Value Model (MVM) described in the next section.

\subsection{Mission Value Model Development}

Ideally, we would have a value model that would allow assessment of alternative architectures directly in terms of 2030 era mission metrics and that could be used in all phases of the study process, as it is important to maintain a clear linkage between the technical performance of future alternative architectures and the missions of national and international importance that they support.

The approach being taken is that we are building in parallel a 2030 era mission value model based on the established NOSIA-II [3] effort within NOAA. This model takes as input the technical performance values determined in the EVM assessment. Hence the two models are linked and can be used to cross-check each other for consistency. Of particular importance, the NOSIA-II model has a full vetted mission model that maps out about 20 Mission Service Areas served by hundreds of discrete data products. This provides an essential overall map allowing identification of the most important sensor data records that play the driving role in multiple mission areas. The NOSIAII model also allows assessment of the relative efficiency of meeting a mission need from a space or ground asset, and so helps identify the most appropriate areas for space observation.

At the time of this writing the NOSIA-II model is being reviewed for how it is likely to change in the 2030s. This review is required to create an MVM relevant to the next generation epoch. Since the MVM will not be fully ready until later in the design cycles, when the review and update is complete, the intent is that it will be used primarily to do a detailed assessment and comparison of particularly promising alternatives identified through screening with the EVM in the later design cycles. The EVM will serve as the primary screening tool, and leading candidates will be assessed more directly with the MVM for impact to mission support. The MVM will allow full assessment of alternative architectures against the mission set before study completion.

\subsection{Integration (and Design) Task}

The central element of the study is the generation and assessment of architecture alternatives. For the purposes of this study an architecture is a definition of a set of functions, a set of physical assets (instruments and their allocation to platforms assigned to specific orbits) assigned to those functions, a concept of operations that shows how the physical assets are employed in time sequence to meet a defined mission, and a set of rules (including policies, standards, protocols and constraints) by which the platforms populate and sustain the resulting constellation. We use the instrument allocations and constellation, in both nominal and probabilistic off-nominal state, to assess performance against the EVM and MVM. We develop costs by analyzing the individual satellites included in the constellation and the construction, storage, and launch history produced by the constellation policies. This requires both the integration of the previous described tasks as well as constellation design and constellation policy development. In addition, there are a number of important study tasks (e.g., industry Request for Information evaluation, formal architecture description writing) that are logically assigned to the same team. We refer to this team as the "Integration Team."

While there are many subtasks within the integration task, we can identify four that are particularly complex and important.

1. Constellation design where we synthesize and examine alternative orbital configurations. We are studying four broad categories: Legacy Continuation, Augmentations to Legacy Continuation, All-Medium Earth Orbit where we use an approach modeled on GPS, and All-LEO where we look at the potential of large swarms of small satellites.

2. Constellation policy design, with particular attention to more complex policies using launchon-need and event-driven orbit adaptation.

3. Constellation level cost analysis, including the impacts of different production levels and use of block design and acquisition.

4. The actual design of satellites given instruments and constellations. This is critical to costing, but must be done in a very rapid and lightweight fashion. The team is utilizing the Aerospace 
Corporation's Concept Design Center capability for this [4], [5].

\subsection{Design Cycles}

The overall process is organized as a series of design cycles. The organization of an architecture study as a series of relatively short end-to-end design cycles has a long history. Rapid turn design cycles are used in civil architecture and urban planning as well as large software projects and major US DoD architecture efforts [6, 7]. There are many advantages to this organization. Probably the most important is that it allows for information that can only be learned in the later phases of integrating a complex design to be fed back into a subsequent cycle. This is particularly important when significant uncertainties exist and it has to be taken as expected that the team will discover user needs, alternatives, or even new CONOPS only after it has generated and assessed one or more complete architectural designs and shown those designs to major stakeholders. It is through these design cycles as well that we evaluate the impact of different architectures on the ground system requirements and capabilities.

\section{SPRWG AND ITS ROLE}

A fact-of-life in this study is that there is no shortage of userneeds, both legacy and emerging. We have a large body of legacy requirements that span from the sensor level through EDRs to missions all of which correspond to established user needs. Nobody is volunteering to give up his or her data or service. On the contrary, all stakeholders can easily think of a wide range of improvements they would like to see in data quality and variety, and can often clearly articulate the mission benefits that might accrue if the data or service could be so improved. In addition to established user needs, there are well recognized needs that are not currently being filled. Finally, the continuing progress in key mission areas, such as numerical weather forecasting, leads to increased demands for data types, resolution, and timeliness. Our problem is much less to identify user needs than it is to 1) organize and prioritize them, and 2) understand the impact of specific observing system capabilities to a range of user needs and to the broader user community overall. One element that is missing is a clear understanding of what changes in the mission space are essentially certain by the 2030s along with what changes are possible but far from certain, and how either type of change will drive, or be driven by, the data available from satellites.

The SPRWG has been set up to manage the interface between stakeholders (widely represented on the SPRWG) and the architecture study team. The SPRWG's primary job will be to determine the details of the tradeable range in the EVM. This will entail looking at projections for mission concepts of operations out to 2030 and assessing where changes to how missions are conducted will be reflected in data quality attributes. Of course, we expect that the result will often be a re-validation of today's requirement, but we also need to know where that expectation is most brought into question and where the areas are that could undergo the largest changes. In addition, the SPRWG will advise the study teams on other aspects of value modeling and relative prioritization of user needs.

\section{CONCLUSIONS}

The NSOSA study is just beginning so any conclusions on future satellite architectures would be premature. Based on the preliminary studies we expect to find it very challenging to balance all mission and programmatic objectives. Our previous studies suggest that there are two key elements to providing improved system performance within budget caps. First is the ability to apply technology developments to create new instrument concepts. Second is to develop more responsive constellation management approaches to simultaneously improve fault tolerance and efficient use of space assets. The NSOSA study will examine alternatives without pre-conceived notions of instrument approaches, assignment to orbits, or approaches to constellation management.

\section{REFERENCES}

[1] Committee on Pre-Milestone $A$ and Early-Phase Systems Engineering: A Retrospective Review and Benefits for Future Air Force Acquisition. Washington, D.C.: The National Academies Press, 2008

[2] Crawley, E., Cameron, B., and Selva, D., System Architecture: Strategy and Product Development for Complex Systems, Pearson, 2016

[3] "NOAA Observing System Integrated Analysis (NOSIA-II), Methodology Report." Silver Spring, MD: National Oceanic and Atmospheric Administration (NOAA), 2015. Retrieved from https://nosc.noaa.gov/tpio/main/nosia main.html.

[4] Aguilar, J. A., \& Dawdy, A. (1999). Scope vs. Detail: The Teams of the Concept Design Center. 2000 IEEE Aerospace Conference Proceedings, (pp. 465-481).

[5] Aguilar, J. A., Dawdy, A. B., \& Law, G. W. (1998). The Aerospace Corporation's Concept Design Center. 8th Annual International Symposium of the International Council on Systems Engineering.

[6] Maier, M., and Rechtin, E., The Art of Systems Architecting, $3^{\text {rd }}$ Edition, CRC Press, 2009.

[7] Di Pietro, D. (2015). A Systems Engineering Approach to Architecture Development. 25 th Annual International Symposium of the International Council on Systems Engineering. 